\title{
Evidence-Based Medicine in Daily Surgical Decision Making: A Survey-Based Comparison between the UK and Germany
}

\author{
Andreas A. Schnitzbauer ${ }^{a} \quad$ Andrea Proneth $^{b} \quad$ Liset Pengel $^{e}$ \\ Jörg Ansorg ${ }^{c}$ Matthias Anthuberd Wolf O. Bechstein ${ }^{a}$ Hans J. Schlitt $^{b}$ \\ Edward K. Geissler ${ }^{b}$ \\ ${ }^{a}$ Department of General and Visceral Surgery, Frankfurt University Hospitals, \\ Goethe University Frankfurt am Main, Frankfurt am Main, ${ }^{b}$ Department of Surgery,

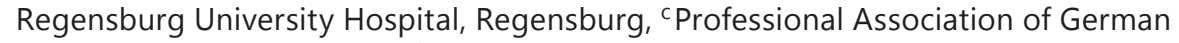 \\ Surgeons (BDC), Berlin, and ${ }^{\mathrm{d} C l i n i c}$ for General, Visceral and Transplant Surgery,

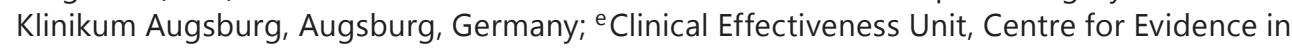 \\ Transplantation, Royal College of Surgeons of England and the London School of Hygiene \\ and Tropical Medicine, University of London, London, UK
}

\section{Key Words}

Evidenced-based medicine · Daily surgical decision making · Survey-based comparison . UK· Germany

\begin{abstract}
Background: Evidence-based medicine (EbM) is a vital part of reasonable and conclusive decision making for clinicians in daily clinical work. To analyze the knowledge and the attitude of surgeons towards EbM, a survey was performed in the UK and Germany. Methods: A webbased questionnaire was distributed via mailing lists from the Royal College of Surgeons of England (RCSE) and the Berufsverband Deutscher Chirurgen (BDC). Our primary aim was to get information about knowledge of EbM amongst German and British surgeons. Results: A total of 549 individuals opened the questionnaire, but only 198 questionnaires were complete and valid for analysis. In total, 40,000 recipients were approached via the mailing lists of the $\mathrm{BDC}$ and RCSE. The response rate was equally low in both countries. On a scale from 1 (unimportant) to 10 (very important), all participants rated EbM as very important for daily clinical decision making $(7.3 \pm 1.9)$ as well as for patients $(7.8 \pm 1.9)$ and the national health system (7.8 \pm 1.9 ). On a scale from 1 (unimportant) to 5 (very important), systematic reviews (4.6 \pm $0.6)$ and randomized controlled trials $(4.6 \pm 0.6)$ were identified as the highest levels of study designs to enhance evidence in medicine. British surgeons considered EbM to be more important in daily clinical work when compared to data from German surgeons (7.9 \pm 1.6 vs. 6.7 $\pm 2.1, \mathrm{p}<0.001)$. Subgroup analysis showed different results in some categories; however, a pattern to explain the differences was not evident. Personal requirements expressed in a free
\end{abstract}


Schnitzbauer et al.: Evidence-Based Medicine in Daily Surgical Decision Making: A

Survey-Based Comparison between the UK and Germany

text field emphasized the results and reflected concerns such as broad unwillingness and lack of interdisciplinary approaches for patients ( $n=59: 25$ in the UK and 34 in Germany). Conclusion: The overall results show that EbM is believed to be important by surgeons in the UK and Germany. However, perception of EbM in the respective health system (UK vs. Germany) may be different. Nonetheless, EbM is an important tool to navigate through daily clinical problems although a discrepancy between the knowledge of theoretical abstract terms and difficulties in implementing EbM in daily clinical work has been detected. The provision of infrastructure, courses and structured education as a permanent instrument will advance the knowledge, application and improvement of EbM in the future.

(c) 2014 S. Karger AG, Basel

\section{Introduction}

Evidence-based medicine (EbM) has been described by Rosenberg and Donald [1] in 1995 as a 'process of systematically finding, appraising, and using contemporaneous research findings as the basis for clinical decisions'. Sackett and colleagues [2,3] defined what evidence is and what it is not by proposing five specific rules: 'firstly, clinical decisions should be based on the best available scientific evidence; secondly, the clinical problem - rather than habits or protocols - should determine the type of evidence to be sought; thirdly, identifying the best evidence means using epidemiological and bio-statistical ways of thinking; fourthly, conclusions derived from identifying and critically appraising evidence are useful only if put into action in managing patients or making health care decisions, and, finally, performance should be constantly evaluated'.

This definition attempts to put every individual patient in the focus of decision making by utilizing the best evidence present, and respects individualization and tailoring. Nonetheless, while substantial use of EbM is readily apparent, the daily clinical behavior of surgeons concerning EbM is not well analyzed [4, 5]. This is particularly relevant to surgery since technical developments and their clinical application often outpace the aspiration for testing its validity in daily clinical use [6].

In December 2011, the question of how surgeons can be convinced to follow results from high-quality evidence-based studies in surgery was raised after a presentation on improving evidence in abdominal and transplant surgery. An idea was proposed to gather information from surgeons about their basic knowledge of EbM in general, and specifically by means of an example from daily surgical care. We developed 5-min surveys including 16 questions, which collected demographic data and included questions and examples relevant to a surgical setting.

To gain a better understanding of how EbM can be better transferred and integrated into daily clinical surgical practice, we performed the survey in the UK and Germany to receive views from two different health care systems. Our primary aim was to get an insight into the surgeons' basic knowledge of EbM and, moreover, to determine if there are differences in knowledge between British and German surgeons.

\section{Methods}

Online Survey, Review and Cooperation

An online survey was generated in German and English which is available at www.q-set.de. The survey was sent to the Centre for Evidence in Transplantation for review and critical analysis of the contents. Finally, the Berufsverband Deutscher Chirurgen (BDC) and the Royal College of Surgeons of England (RCSE) agreed to post the survey on their respective homepages and distribute the online link via their mailing list in their 
Schnitzbauer et al.: Evidence-Based Medicine in Daily Surgical Decision Making: A Survey-Based Comparison between the UK and Germany

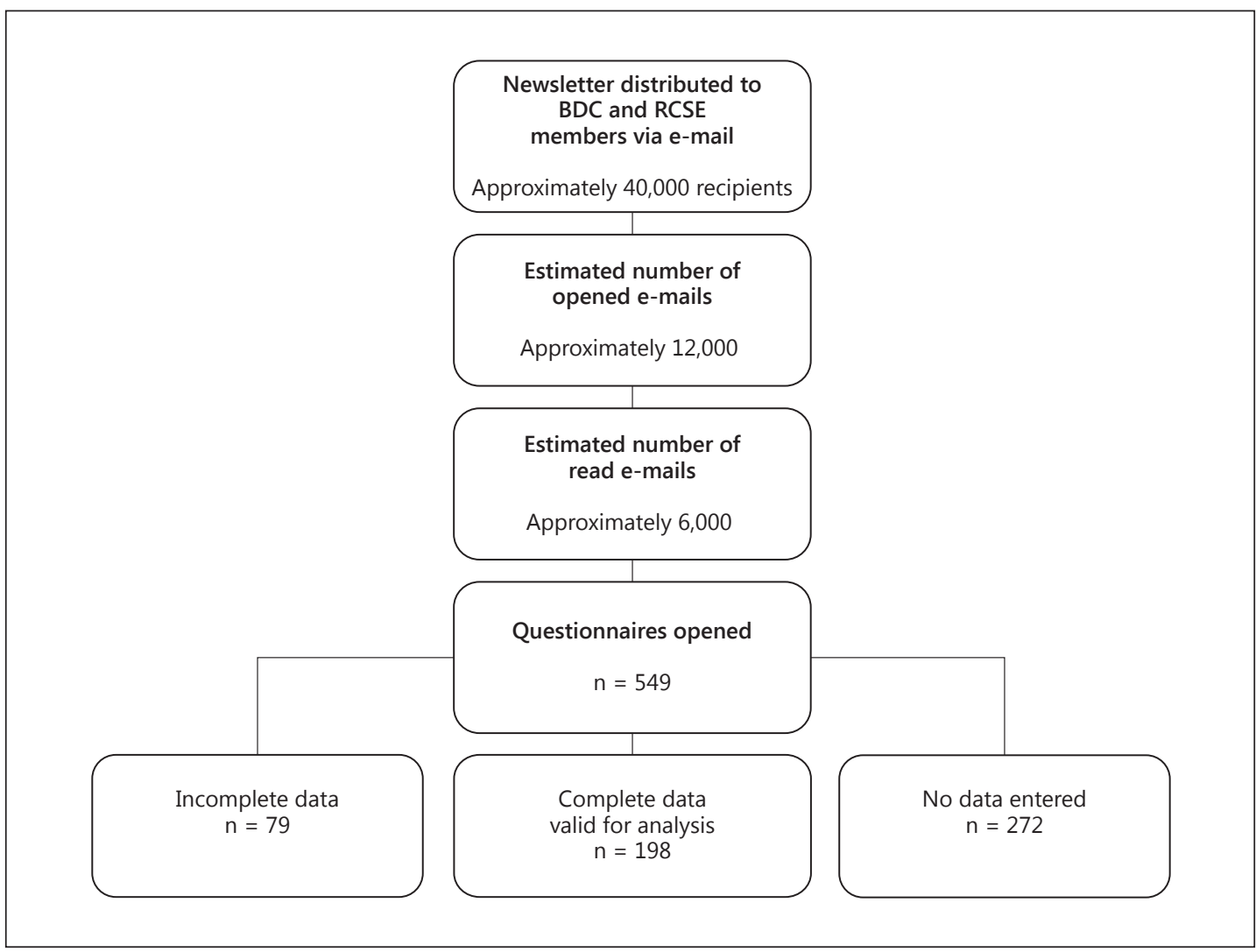

Fig. 1. Flowchart explaining the response rate.

periodic online newsletters. The survey was launched online on May 15, 2012, with a reminder being sent to members in September 2012; the database was finally closed on December 1, 2012. Demographic data (gender, age, position, supply level of hospital) were collected in the first four questions. Then, the relevance of EbM regarding daily work, the national health system and patients was raised. Knowledge of EbM was tested by asking questions about a common clinical procedure, namely laparoscopic cholecystectomy. Moreover, knowledge about different tools of EbM or providing evidence in medicine, such as databases or quality of studies, was retrieved from participants. Finally, participants could express their wishes and requirements to improve knowledge in EbM.

\section{Statistics}

Statistical analyses were performed using Sigma Stat 11.0 (Systat Software Inc., Richmond, Calif., USA). Data are given as means with standard deviation, and the median with minimum and maximum was calculated. T tests were performed to detect significant differences between groups. A p value $<0.05$ was regarded to reflect statistically significant differences.

\section{Results}

\section{Response Rate}

The mailing lists of the BDC and the RCSE contained approximately 40,000 e-mails. Both institutions estimated that the e-mails would typically be opened by approximately $30 \%$ of the recipients. Then, we estimated that approximately $50 \%$ would read the newsletter. The survey was announced on the first page and was highlighted to attract a high number of readers. Finally, a total of 549 questionnaires were opened via the link provided in the news- 
Table 1. Participants' overview

\begin{tabular}{|c|c|c|c|c|}
\hline \multicolumn{2}{|l|}{ Categories } & \multirow{2}{*}{$\begin{array}{l}\text { UK } \\
(\mathrm{n}=96) \\
19(20) \\
77(80)\end{array}$} & \multirow{2}{*}{$\begin{array}{c}\text { Germany } \\
\text { (n = 102) }\end{array}$} & \multirow{2}{*}{$\begin{array}{l}\begin{array}{l}\text { Total } \\
(\mathrm{n}=198)\end{array} \\
\begin{array}{r}43(22) \\
155(78)\end{array}\end{array}$} \\
\hline Gender & $\begin{array}{l}\text { female } \\
\text { male }\end{array}$ & & & \\
\hline & subtotal & 96 & 102 & 198 \\
\hline \multirow[t]{5}{*}{ Age } & $\begin{array}{l}25-30 \text { years } \\
31-35 \text { years }\end{array}$ & $\begin{array}{c}8(8) \\
17(18)\end{array}$ & $\begin{array}{l}19(19) \\
13(13)\end{array}$ & $\begin{array}{l}27(14) \\
30(15)\end{array}$ \\
\hline & $36-40$ years & $10(10)$ & $23(23)$ & $33(17)$ \\
\hline & $41-50$ years & $19(20)$ & $25(25)$ & $44(22)$ \\
\hline & $>50$ years & $42(44)$ & $22(21)$ & $64(32)$ \\
\hline & subtotal & 96 & 102 & 198 \\
\hline \multirow[t]{6}{*}{ Position } & resident & $18(19)$ & $32(31)$ & $40(20)$ \\
\hline & fellow & $11(11)$ & $18(18)$ & $29(15)$ \\
\hline & consultant & $51(53)$ & $29(28)$ & $80(40)$ \\
\hline & director/head of program or clinic & $7(7)$ & $15(15) 0$ & $22(11) 0$ \\
\hline & other & $9(9)$ & $8(8)$ & $17(9)$ \\
\hline & subtotal & 96 & 102 & 198 \\
\hline \multirow[t]{3}{*}{ Service level } & basic supply & $1(1)$ & $17(17)$ & $18(9)$ \\
\hline & secondary hospital & 47 (49) & $24(24)$ & 71 (36) \\
\hline & $\begin{array}{l}\text { tertiary referral hospital (highly specialized } \\
\text { and university clinics) }\end{array}$ & $48(50)$ & $61(60) 0$ & $109(55)$ \\
\hline
\end{tabular}

Values in parentheses are percentages. For Germany, positions and service levels have been transferred as approximation: resident = Arzt in Weiterbildung; fellow = Facharzt; consultant = Oberarzt; director $/$ head of program or clinic = Chefarzt; basic supply = Grund- und Regelversorgung; secondary hospital = Schwerpunktversorgung; tertiary referral hospital = Maximalversorgung.

letter, but only 198 questionnaires were completed and valid for analysis. Figure 1 depicts a response rate flow chart for the survey.

\section{Gender, Age, Position and Service Level}

Table 1 gives an overview of the participants' gender, age, and position and the service level of both countries. Importantly, there were similar numbers of completed questionnaires collected from Germany $(n=102)$ and the UK $(n=96)$.

\section{Overall Results}

All participants rated the usefulness of EbM in their daily clinical work with a mean of 7.3 \pm 1.9 points (range: $1=$ unimportant to $10=$ very important). The usefulness of the EbM for the national health system and for patients was given $7.8 \pm 1.9$ points. Based on a clinically relevant example, the participants were asked to give their opinion as to why laparoscopic cholecystectomy is considered as the standard technique. They had to validate the following statements: (1) laparoscopic cholecystectomy is safer than the open technique $(4.0 \pm 2.5$ points), (2) patients ask for it ( $6.8 \pm 2.6$ points), and (3) it is more cost-effective for the health system ( $5.6 \pm 2.8$ points); the answer could be valued from 1 (wrong) to 10 (correct). Finally, the participants were asked about which study designs expand evidence in medicine significantly. The highest ratings were obtained for systematic reviews and meta-analyses ( $4.6 \pm 0.6$ points), randomized controlled trials (RCTs; $4.5 \pm 0.6$ points) and systematic reviews of 
Schnitzbauer et al.: Evidence-Based Medicine in Daily Surgical Decision Making: A Survey-Based Comparison between the UK and Germany

Table 2. Comparison of the British and German surgeons' opinions regarding the utility of EbM, the role of laparoscopic cholecystectomy representing a daily clinically relevant example, and the role of study quality in increasing evidence in medicine

\begin{tabular}{|c|c|c|c|c|c|c|c|}
\hline & \multicolumn{3}{|l|}{ UK $(n=96)$} & \multicolumn{3}{|c|}{ Germany $(n=102)$} & \multirow{2}{*}{$\begin{array}{l}\mathrm{p} \\
\text { value }\end{array}$} \\
\hline & mean \pm SD & median & range & mean \pm SD & median & range & \\
\hline \multicolumn{8}{|l|}{ How would you rate the utility of EbM ... ${ }^{a}$} \\
\hline ... regarding daily work? & $7.9 \pm 1.6$ & 8 & $3-10$ & $6.7 \pm 2.1$ & 8 & $1-10$ & $<0.001$ \\
\hline ... regarding the national health system? & $7.8 \pm 1.9$ & 8 & $2-10$ & $7.4 \pm 2.1$ & 8 & $1-10$ & 0.255 \\
\hline ... for patients? & $8.1 \pm 1.6$ & 8 & $2-10$ & $7.5 \pm 2.0$ & 8 & $1-10$ & 0.065 \\
\hline \multicolumn{8}{|c|}{ Laparoscopic cholecystectomy is today's standard surgical procedure because ...b } \\
\hline ... it is safer & $4.9 \pm 2.3$ & 5 & $1-10$ & $3.2 \pm 2.4$ & 5 & $1-9$ & $<0.001$ \\
\hline ... patients ask for it & $5.8 \pm 2.5$ & 5 & $1-10$ & $7.7 \pm 2.3$ & 5 & $1-10$ & $<0.001$ \\
\hline ... it is more cost-effective & $6.7 \pm 2.4$ & 7 & $1-10$ & $4.6 \pm 2.9$ & 7 & $1-10$ & $<0.001$ \\
\hline \multicolumn{8}{|c|}{ Which types of studies expand evidence in medicine significantly? } \\
\hline Meta-analyses & $4.6 \pm 0.6$ & 5 & $2-5$ & $4.5 \pm 0.6$ & 5 & $2-5$ & 0.56 \\
\hline RCTs & $4.4 \pm 0.6$ & 4 & $3-5$ & $4.6 \pm 0.5$ & 4 & $3-5$ & 0.01 \\
\hline Systematic reviews & $3.7 \pm 0.7$ & 4 & $2-5$ & $3.4 \pm 0.8$ & 4 & $1-5$ & 0.001 \\
\hline Individual cohort studies & $2.9 \pm 0.8$ & 3 & $1-5$ & $2.7 \pm 0.8$ & 3 & $1-5$ & 0.016 \\
\hline Case control studies & $2.9 \pm 0.8$ & 3 & $1-5$ & $2.7 \pm 1.0$ & 3 & $1-5$ & 0.161 \\
\hline Case series & $2.4 \pm 1.0$ & 2 & $1-5$ & $2.2 \pm 1.0$ & 2 & $1-5$ & 0.092 \\
\hline Expert opinions & $2.5 \pm 1.2$ & 2 & $1-5$ & $2.3 \pm 1.1$ & 2 & $1-5$ & 0.182 \\
\hline
\end{tabular}

a $1=$ unimportant, $10=$ very important; ${ }^{\mathrm{b}} 1=$ wrong, $^{\mathrm{a}} 10=$ correct; $^{\mathrm{c}} 1=$ unimportant, $^{\mathrm{a}}=$ very important.

cohort studies ( $3.6 \pm 0.8$ points). The answer's importance could be valued from 1 (unimportant) to 5 (very important).

\section{Comparing EbM in the UK and Germany}

For the secondary analysis, the results from the participants in the UK $(n=96)$ and Germany $(n=102)$ were compared (table 2). Significantly, more British surgeons thought that EbM was very useful in daily clinical work compared to German surgeons (7.9 \pm 1.6 vs. $6.7 \pm 2.1, \mathrm{p}<0.001$ ). Additionally, there were completely different viewpoints concerning safety, patient preference and cost-effectiveness of laparoscopic cholecystectomy compared to open interventions. On a scale from 0 to 10, British (vs. German) surgeons more strongly believed that laparoscopic cholecystectomy is the standard surgical procedure because it is safer $(4.9 \pm 2.3$ vs. $3.2 \pm 2.4, \mathrm{p}<0.001)$ and more cost-effective $(6.7 \pm 2.4$ vs. $4.6 \pm 2.9, \mathrm{p}<$ 0.001). In contrast, German (vs. British) surgeons more strongly believed that laparoscopic cholecystectomy is the standard procedure because patients ask for it ( $5.8 \pm 2.5$ vs. $7.7 \pm 2.3$, $\mathrm{p}<0.001)$. There were very minor but significant differences regarding the study designs that are considered to significantly expand evidence (table 2).

\section{EbM Comparing Different Ages, Gender, Positions and Service Levels}

Subgroup analyses were performed to explore the detected differences in a more detailed manner. However, the results were nearly the same throughout all analyzed British and German subgroups for age, gender, position and supply level (secondary vs. tertiary) of the hospital (data not shown). Analysis of combined subgroups from both countries (female vs. male, $\leq 40$ vs. $>40$ years, in education vs. leaders, basic/secondary supply vs. tertiary supply) revealed the largest differences for surgeons working in basic and secondary supply hospitals when compared to surgeons in tertiary supply hospitals. Here, 7 of 13 questions that were 
Schnitzbauer et al.: Evidence-Based Medicine in Daily Surgical Decision Making: A Survey-Based Comparison between the UK and Germany

Table 3. Comparison of demographic subgroups regarding the utility of EbM, the role of laparoscopic cholecystectomy representing a daily clinically relevant example, and the value of study quality to increase evidence in medicine

\begin{tabular}{|c|c|c|c|c|c|c|c|c|c|c|c|c|}
\hline & $\begin{array}{l}\text { Female } \\
(n=43)\end{array}$ & $\begin{array}{l}\text { Male } \\
(n=155)\end{array}$ & $\begin{array}{l}\mathrm{p} \\
\text { value }\end{array}$ & $\begin{array}{l}\leq 40 \text { years } \\
(\mathrm{n}=90)\end{array}$ & $\begin{array}{l}>40 \text { years } \\
(n=108)\end{array}$ & $\begin{array}{l}\mathrm{p} \\
\text { value }\end{array}$ & $\begin{array}{l}\text { Trainees } \\
(\mathrm{n}=79)\end{array}$ & $\begin{array}{l}\text { Consultants } \\
(\mathrm{n}=102)\end{array}$ & $\begin{array}{l}\mathrm{p} \\
\text { value }\end{array}$ & $\begin{array}{l}\text { Basic/ } \\
\text { secondary } \\
(\mathrm{n}=89)\end{array}$ & $\begin{array}{l}\text { Tertiary } \\
\text { hospitals } \\
(n=109)\end{array}$ & $\begin{array}{l}\mathrm{p} \\
\text { value }\end{array}$ \\
\hline \multicolumn{13}{|c|}{ How would you rate the utility of EbM ... a } \\
\hline ... regarding daily work? & $7(3-10)$ & $8(1-10)$ & 0.333 & $7(1-10)$ & $8(3-10)$ & 0.088 & $7(1-10)$ & $8(3-10)$ & 0.009 & $8(1-10)$ & $8(3-10)$ & 0.662 \\
\hline $\begin{array}{l}\text {... regarding the national } \\
\text { health system? }\end{array}$ & $9(3-10)$ & $8(1-10)$ & 0.033 & $8(1-10)$ & $8(1-10)$ & 0.062 & $8(1-10)$ & $8(3-10)$ & 0.466 & $8(1-10)$ & $8(2-10)$ & 0.443 \\
\hline ... for patients? & $9(3-10)$ & $8(1-10)$ & 0.086 & $8(1-10)$ & $8(3-10)$ & 0.336 & $8(1-10)$ & $8(1-10)$ & 0.576 & $8(1-10)$ & $8(1-10)$ & 0.464 \\
\hline \multicolumn{13}{|c|}{ Laparoscopic cholecystectomy is today's standard surgical procedure because ... } \\
\hline$\ldots$ it is safer & $4(1-9)$ & $4(1-10)$ & 0.546 & $4(1-10)$ & $4(1-10)$ & 0.203 & $3(1-10)$ & $4(1-10)$ & 0.544 & $4(1-10)$ & $2(1-9)$ & 0.002 \\
\hline ... patients ask for it & $7(1-10)$ & $8(1-10)$ & 0.05 & $8(1-10)$ & $7(1-10)$ & 0.553 & $7(1-10)$ & $7(1-10)$ & 0.914 & $7(1-10)$ & $8(1-10)$ & 0.011 \\
\hline ... it is more cost-effective & $6(1-10)$ & $5(1-10)$ & 0.212 & $5(1-10)$ & $5(1-10)$ & 0.780 & $6(1-10)$ & $5(1-10)$ & 0.344 & $5(1-10)$ & $4(1-10)$ & 0.009 \\
\hline \multicolumn{13}{|c|}{ Which types of studies expand evidence in medicine significantly? } \\
\hline Meta-analyses & $5(2-5)$ & $5(2-5)$ & 0.454 & $5(2-5)$ & $5(2-5)$ & 0.049 & $5(2-5)$ & $5(2-5)$ & 0.037 & $5(2-5)$ & $5(2-5)$ & 0.550 \\
\hline RCTs & $5(3-5)$ & $5(3-5)$ & 0.282 & $5(3-5)$ & $4(3-5)$ & 0.003 & $5(3-5)$ & $4(3-5)$ & 0.028 & $4(3-5)$ & $5(3-5)$ & 0.001 \\
\hline Systematic reviews & $3(1-4)$ & $4(1-5)$ & 0.249 & $4(1-5)$ & $4(1-5)$ & 0.425 & $4(1-5)$ & $4(2-5)$ & 0.523 & $4(1-5)$ & $3(1-5)$ & 0.112 \\
\hline Individual cohort studies & $3(1-4)$ & $3(1-5)$ & 0.921 & $3(1-5)$ & $3(1-4)$ & 0.699 & $3(1-5)$ & $3(1-4)$ & 0.495 & $3(1-4)$ & $3(1-5)$ & 0.024 \\
\hline Case control studies & $3(1-5)$ & $3(1-5)$ & 0.370 & $3(1-5)$ & $3(1-5)$ & 0.435 & $3(1-5)$ & $3(1-4)$ & 0.324 & $3(1-5)$ & $2(1-4)$ & $<0.001$ \\
\hline Case series & $2(1-4)$ & $2(1-5)$ & 0.495 & $2(1-5)$ & $2(1-5)$ & 0.247 & $2(1-5)$ & $2(1-4)$ & 0.996 & $2(1-5)$ & $2(1-5)$ & 0.005 \\
\hline Expert opinions & $2(1-5)$ & $2(1-5)$ & 0.355 & $2(1-5)$ & $3(1-5)$ & 0.038 & $2(1-5)$ & $2(1-5)$ & 0.165 & $3(1-5)$ & $2(1-4)$ & 0.062 \\
\hline
\end{tabular}

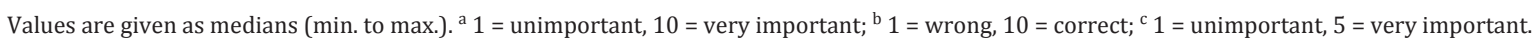

asked revealed significant differences (table 3). Both, the participants from basic supply hospital as well as the participants from secondary/tertiary supply hospitals rated EbM with the same importance for daily work, patients and the national health systems. Views on safety, patient preferences and cost-effectiveness of laparoscopic cholecystectomies, however, differed significantly. Moreover, their perception of study quality importance regarding the influence on generating evidence was significantly different.

\section{Personal Requirements and Worries of Surgeons Concerning EbM}

Free text fields at the end of the survey were available for the participants to express their personal concerns and requirements concerning a reasonable and successful application of EbM. A total of 172 answers were received, 86 from Germany and 86 from the UK. There were various, though similar comments, from both German and British surgeons. Some participants gave multiple comments. Many expressed their concern that hierarchies in hospitals are still too strict. They also expressed their concern that teaching in EbM is still not adequate during surgical training. Additionally, there is a broad unwillingness and lack of interdisciplinary approaches for patients ( $n=59: 25$ in the UK and 34 in Germany). Time ( $n=31)$ and financial aspects $(n=20)$ were also identified as critical factors, which included lack of time for reading and literature research as well as workload in the clinic combined with administrative pressure. Lack of evidence for interventions $(n=14)$, lack of guidelines $(n=5)$, political reasons $(n=6)$, overload of sources, such as search tools and databases $(n=7)$, and patient preferences for a specific therapy $(n=4)$ were less frequently mentioned. A total of 23 participants did not mention any concerns. Finally, 15 participants explained that there is often a lack of EbM and that individualized therapies for patients are performed without established guidelines.

\section{Participant Requirements}

Finally, there was a broad consensus amongst the participants that more teaching of EbM in the form of special congress topics, online information and continuing medical education is absolutely necessary to establish and deepen knowledge of this issue in surgery. 
Schnitzbauer et al.: Evidence-Based Medicine in Daily Surgical Decision Making: A

Survey-Based Comparison between the UK and Germany

\section{Discussion}

EbM is recognized as a very important subject amongst surgeons in Germany and the UK. The median rating of surgeons concerning the utility of EbM for daily work decisions, the national health system and for patients was high. Moreover, the participants adequately rated the study designs that provide higher levels of evidence. However, it continues to be an abstract field which although recognized as important is not always used in daily clinical practice [6]. For instance, there was a common discrepancy between existing evidence and a long practiced experience by senior surgeons, evident in our survey through raised concerns (30\% of the participants) in the open comment section.

Although perceptions of EbM deliver high scores and quite similar results for both British and German surgeons, the clinical example of the utility of laparoscopic cholecystectomy revealed a somewhat different perception, representing one of the most commonly performed procedures worldwide. More specifically, British surgeons in comparison to German surgeons were of the opinion that laparoscopic cholecystectomy is safer than open cholecystectomy. Literature research identified three meta-analyses from the Cochrane database where open cholecystectomy and mini-incision cholecystectomy are compared to laparoscopic cholecystectomy [7-9]. There were no differences when comparing safety, in terms of the complication rates, between the open and laparoscopic techniques. However, it is interesting that complication rates were higher in high-quality RCTs (17\%) compared to retrospective cohort studies (5\%). Laparoscopic cholecystectomy is associated with a faster recovery and discharge of patients. Whilst the costs for surgery alone are higher for laparoscopic cholecystectomy, some authors argue that the overall costs to the health care and economic systems are reduced by shorter clinic stays that lead in many cases to patients returning back to work faster [7-17]. Regarding patient preferences, there is no evidence in the literature that patients ask their surgeon for laparoscopic surgery. Therefore, the existing evidence shows no surgical superiority of laparoscopic cholecystectomy in terms of safety. The evidence supporting laparoscopic cholecystectomy is related to the shorter postoperative clinical course but not to the surgeon's beliefs regarding patient preference.

Personal comments at the end of the survey helped to expand opinions restricted by the categories selected in the survey. Participants used various arguments to express their requirements and restrictions associated with EbM involvement. Reasons highlighted for rejecting EbM included strict hierarchies, lack of EbM training, missing interdisciplinary approaches, time, absence of financial means, lack of evidence and guidelines, and resource overload (e.g. search tools or databases).

This leads to the discussion of possible solutions. First and foremost, the integration of EbM in education must be constant from the early days of medical school to daily clinical work in every particular field of surgery. A good example of the integration of EbM in societies is the syllabus for different certified surgical examinations for the European Union of Medical Specialists of Surgery and European Board of Surgery. One of the requirements is the ability to interpret and analyze data from a variety of papers provided for the examination. Secondly, certification of centers for a special focus, such as pancreas, colorectal, or endocrine cancer promotes interdisciplinary approaches, typically through transparent case evaluation boards that pool current evidence [18-20]. Thirdly, the scientific infrastructure of surgical societies should be improved. For instance, in Germany, the German Surgical Society (DGCH) supports and funds a study center in Heidelberg charged with the task of developing and conducting clinical trials in surgery $[4,5,21,22]$. Fourthly, access to high-level evidence for busy clinicians should be facilitated. To this end, the Centre for Evidence in Transplantation (www. transplantevidence.com) has established the Transplant Library - a specialist database of all RCTs and good-quality systematic reviews in solid organ transplantation [23]. The Library 
can be used without professional search skills and gives quick and easy access to high-level evidence.

Comments from the respondents raised the issue whether a critical review of established surgical procedures that have been successfully applied for more than 150 years is needed. In general, while challenging these basic methods is perhaps futile, single aspects of certain surgical procedures or treatments can expose clinical insecurities and should therefore be clarified by randomized clinical trials. Thus, a categorization of surgical techniques and the relevance to explore more evidence might be helpful: (i) classic surgical techniques that have been described in textbooks should be regarded as the standard treatment of care; (ii) contemporary surgical innovations should be regarded as the standard treatment of care today (e.g. laparoscopic cholecystectomy, continuously evolving surgical techniques, and natural orifice transluminal endoscopic surgery, single incision laparoscopic surgery or associating liver partition with portal vein ligation for staged hepatectomy in liver resection [24]); (iii) diseases with a lack of guidelines refer to standard treatments and classifications that exist without sufficient and convincing evidence from clinical trials (e.g. sigmoid diverticulitis, suture or stapling of loop ileostomy closure, clipping or sutures in thyroid surgery, immunosuppression in patients with renal impairment or hepatocellular carcinoma after liver transplantation [25-33]), and (iv) complex treatment decisions in interdisciplinary oncologic settings that still reveal clinical insecurity and need clarification with clinical trials (e.g. neoadjuvant and adjuvant therapy strategies in colorectal liver metastases), but also warrant an individualized and tailored therapy.

A major limitation of our study is the low response rate to the survey. Although distributed and announced via two major professional societies highlighting the survey in their regular newsletter, the response rate was low. This may be a problem regarding internet-based surveys in general, since the request to participate is not made personally to every single person and potential participants are overloaded with requests to participate in surveys. The results in this survey may be prone to some bias because persons with a positive view of EbM may have been more likely to participate. Nonetheless, opinion polls in political discussions face similar hurdles but do tend to deliver fairly exact pictures with small polling numbers. If we are sincere in our efforts to improve EbM, more academic surgeons should feel obligated to participate in such efforts.

In conclusion, our survey indicates that EbM is seen as an important tool to navigate through daily clinical insecurities. However, surgeons often underutilize structured state-ofthe-art evidence resources. Efforts need to be intensified to properly mentor involved surgical personnel through local programs and structured education systems. National study centers like the Study Centre of the DGCH in Heidelberg are also an instrument to foster and establish a culture of medicine on the highest level of obtainable evidence.

\section{Acknowledgements}

We deeply thank all participants in the UK and Germany for filling out the questionnaire and for their very personal comments that helped us to unveil the requirements in surgery-related EbM in both countries. Additionally, we thank Marius Müller for providing the national platform in the UK for the distribution of the e-mails. Peter Morris functioned as adviser in the development of the questionnaire.

\section{Disclosure Statement}

The authors have no conflicts of interest to disclose. 
Schnitzbauer et al.: Evidence-Based Medicine in Daily Surgical Decision Making: A Survey-Based Comparison between the UK and Germany

\section{References}

1 Rosenberg W, Donald A: Evidence based medicine: an approach to clinical problem-solving. BMJ 1995;310: 1122-1126.

2 Sackett DL, Rosenberg WM, Gray JA, Haynes RB, Richardson WS: Evidence based medicine: what it is and what it isn't. BMJ 1996;312:71-72.

3 Davidoff F, Haynes B, Sackett D, Smith R: Evidence based medicine. BMJ 1995;310:1085-1086.

4 Diener MK, Knebel P, Fink C, Dörr-Harim C, Rossion I, Werner J, et al: Clinical trials in surgery. On the way towards evidence-based surgery (in German). Chirurg 2012;83:315-318.

5 Diener MK, Wolff RF, von Elm E, Rahbari NN, Mavergames C, Knaebel H-P, et al: Can decision making in general surgery be based on evidence? An empirical study of Cochrane Reviews. Surgery 2009;146:444-461.

6 Schwenk W, Haase 0, Müller JM: Changing surgical therapy because of clinical studies? (in German). Zentralbl Chir 2002;127:258-264.

7 Keus F, de Jong JAF, Gooszen HG, van Laarhoven CJHM: Laparoscopic versus open cholecystectomy for patients with symptomatic cholecystolithiasis. Cochrane Database Syst Rev 2006;4:CD006231.

-8 Keus F, de Jong JAF, Gooszen HG, van Laarhoven CJHM: Laparoscopic versus small-incision cholecystectomy for patients with symptomatic cholecystolithiasis. Cochrane Database Syst Rev 2006;4:CD006229.

-9 Keus F, Gooszen HG, van Laarhoven CJ: Open, small-incision, or laparoscopic cholecystectomy for patients with symptomatic cholecystolithiasis. An overview of Cochrane Hepato-Biliary Group reviews. Cochrane Database Syst Rev 2010;1:CD008318.

10 Barkun AN, Barkun JS, Sampalis JS, Caro J, Fried GM, Meakins JL, et al: Costs and effectiveness of extracorporeal gallbladder stone shock wave lithotripsy versus laparoscopic cholecystectomy. A randomized clinical trial. McGill Gallstone Treatment Group. Int J Technol Assess Health Care 1997;13:589-601.

$\checkmark 11$ Bass EB, Pitt HA, Lillemoe KD: Cost-effectiveness of laparoscopic cholecystectomy versus open cholecystectomy. Am J Surg 1993;165:466-471.

12 Bosch F, Wehrman U, Saeger H-D, Kirch W: Laparoscopic or open conventional cholecystectomy: clinical and economic considerations. Eur J Surg Acta Chir 2002;168:270-277.

13 Fajardo R, Valenzuela JI, Olaya SC, Quintero G, Carrasquilla G, Pinzón CE, et al: Cost-effectiveness of laparoscopic versus open cholecystectomy (in Spanish). Biomedica 2011;31:514-524.

14 Fullarton GM, Darling K, Williams J, MacMillan R, Bell G: Evaluation of the cost of laparoscopic and open cholecystectomy. Br J Surg 1994;81:124-126.

$\longrightarrow 15$ Kelley JE, Burrus RG, Burns RP, Graham LD, Chandler KE: Safety, efficacy, cost, and morbidity of laparoscopic versus open cholecystectomy: a prospective analysis of 228 consecutive patients. Am Surg 1993;59:23-27.

16 Keskin A: Is laparoscopic cholecystectomy cheaper? Surg Laparosc Endosc Percutan Tech 2005;15:191-194; discussion 194.

17 Kesteloot K, Penninckx F: The costs and effects of open versus laparoscopic cholecystectomies. Health Econ 1993;2:303-312.

18 Keating NL, Landrum MB, Lamont EB, Bozeman SR, Shulman LN, McNeil BJ: Tumor boards and the quality of cancer care. J Natl Cancer Inst 2013;105:113-121.

19 Bell RH: National curricula, certification and credentialing. Surgeon 2011;9(suppl 1):S10-S11.

20 Weikert S, Baumunk D, Stephan C, Cash H, Jahnke K, Steiner U, et al: Introduction of interdisciplinary prostate cancer centers based on the recommendations of the German Cancer Society. A cost-benefit analysis 3 years after accreditation (in German). Urologe A 2011;50:1083-1088.

-21 Fink C, Keck T, Rossion I, Weitz J, Diener MK, Büchler MW, et al: Contribution of the Study Center of the German Surgical Society to evidence based surgery (in German). Chirurg 2011;82:1109-1115.

-22 Schiessling S, Diener MK, Post S, Büchler MW, Seiler CM: Clinical trials in surgery - health care research of the future? (in German). Zentralbl Chir 2011;136:87-89.

-23 Pengel L, Morris P: The transplant library of randomized controlled trials and systematic reviews. Transplantation 2011;92:613-616.

24 Schnitzbauer AA, Lang SA, Goessmann H, Nadalin S, Baumgart J, Farkas SA, et al: Right portal vein ligation combined with in situ splitting induces rapid left lateral liver lobe hypertrophy enabling 2-staged extended right hepatic resection in small-for-size settings. Ann Surg 2012;255:405-414.

-25 Hackert T, Bruckner T, Dörr-Harim C, Diener MK, Knebel P, Hartwig W, et al: Pylorus resection or pylorus preservation in partial pancreatico-duodenectomy (PROPP study): study protocol for a randomized controlled trial. Trials 2013;14:44.

-26 Löffler T, Rossion I, Bruckner T, Diener MK, Koch M, von Frankenberg M, et al: HAnd Suture Versus STApling for Closure of Loop Ileostomy (HASTA Trial): results of a multicenter randomized trial (DRKS00000040). Ann Surg 2012;256:828-835; discussion 835-836.

27 Diener MK, Seiler CM, von Frankenberg M, Rendel K, Schüle S, Maschuw K, et al: Vascular clips versus ligatures in thyroid surgery - results of a multicenter randomized controlled trial (CLIVIT Trial). Langenbecks Arch Surg 2012;397:1117-1126.

-28 Schnitzbauer AA, Schlitt HJ, Geissler EK: Influence of immunosuppressive drugs on the recurrence of hepatocellular carcinoma after liver transplantation: a gap between basic science and clinical evidence. Transplantation 2011;91:1173-1176. 
29 Schnitzbauer AA, Doenecke A, Sothmann JL, Loss M, Farkas SA, Hartl J, et al: Improved outcome after 'bottomup' immunosuppression in liver transplant recipients with preoperative renal impairment. Eur Surg Res 2010;45:356-367.

-30 Schnitzbauer AA, Scherer MN, Rochon J, Sothmann J, Farkas SA, Loss M, et al: Study protocol: a pilot study to determine the safety and efficacy of induction-therapy, de novo MPA and delayed mTOR-inhibition in liver transplant recipients with impaired renal function. PATRON-study. BMC Nephrol 2010;11:24.

-31 Schnitzbauer AA, Zuelke C, Graeb C, Rochon J, Bilbao I, Burra P, et al: A prospective randomised, open-labeled, trial comparing sirolimus-containing versus mTOR-inhibitor-free immunosuppression in patients undergoing liver transplantation for hepatocellular carcinoma. BMC Cancer 2010;10:190.

32 Glockzin G, Rochon J, Arnold D, Lang SA, Klebl F, Zeman F, et al: A prospective multicenter phase II study evaluating multimodality treatment of patients with peritoneal carcinomatosis arising from appendiceal and colorectal cancer: the COMBATAC trial. BMC Cancer 2013;13:67.

33 Stein A, Glockzin G, Wienke A, Arnold D, Edelmann T, Hildebrandt B, et al: Treatment with bevacizumab and FOLFOXIRI in patients with advanced colorectal cancer: presentation of two novel trials (CHARTA and PERIMAX) and review of the literature. BMC Cancer 2012;12:356. 